University of Nebraska - Lincoln

DigitalCommons@University of Nebraska - Lincoln

U.S. Environmental Protection Agency Papers

U.S. Environmental Protection Agency

2007

\title{
PBPK models in risk assessment-A focus on chloroprene
}

Robert S. DeWoskin

U.S.EPA, dewoskin.rob@epa.gov

Follow this and additional works at: https://digitalcommons.unl.edu/usepapapers

DeWoskin, Robert S., "PBPK models in risk assessment-A focus on chloroprene" (2007). U.S. Environmental Protection Agency Papers. 163.

https://digitalcommons.unl.edu/usepapapers/163

This Article is brought to you for free and open access by the U.S. Environmental Protection Agency at DigitalCommons@University of Nebraska - Lincoln. It has been accepted for inclusion in U.S. Environmental Protection Agency Papers by an authorized administrator of DigitalCommons@University of Nebraska - Lincoln. 


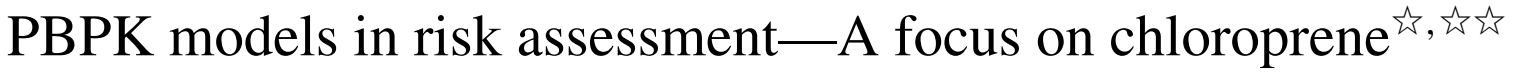

\author{
Robert S. DeWoskin* \\ US EPA/NCEA (National Center for Environmental Assessment), Mail Drop B243-01, Research Triangle Park, NC 27711, United States
}

Available online 8 February 2007

\begin{abstract}
Mathematical models are increasingly being used to simulate events in the exposure-response continuum, and to support quantitative predictions of risks to human health. Physiologically based pharmacokinetic (PBPK) models address that portion of the continuum from an external chemical exposure to an internal dose at a target site. Essential data needed to develop a PBPK model include values of key physiological parameters (e.g., tissue volumes, blood flow rates) and chemical specific parameters (rate of chemical absorption, distribution, metabolism, and elimination) for the species of interest. PBPK models are commonly used to: (1) predict concentrations of an internal dose over time at a target site following external exposure via different routes and/or durations; (2) predict human internal concentration at a target site based on animal data by accounting for toxicokinetic and physiological differences; and (3) estimate variability in the internal dose within a human population resulting from differences in individual pharmacokinetics. Himmelstein et al. [M.W. Himmelstein, S.C. Carpenter, P.M. Hinderliter, Kinetic modeling of beta-chloroprene metabolism. I. In vitro rates in liver and lung tissue fractions from mice, rats, hamsters, and humans, Toxicol. Sci. 79 (1) (2004) 18-27; M.W. Himmelstein, S.C. Carpenter, M.V. Evans, P.M. Hinderliter, E.M. Kenyon, Kinetic modeling of beta-chloroprene metabolism. II. The application of physiologically based modeling for cancer dose response analysis, Toxicol. Sci. 79 (1) (2004) 28-37] developed a PBPK model for chloroprene (2-chloro-1,3-butadiene; CD) that simulates chloroprene disposition in rats, mice, hamsters, or humans following an inhalation exposure. Values for the CD-PBPK model metabolic parameters were obtained from in vitro studies, and model simulations compared to data from in vivo gas uptake studies in rats, hamsters, and mice. The model estimate for total amount of metabolite in lung correlated better with rodent tumor incidence than did the external dose. Based on this PBPK model analytical approach, Himmelstein et al. [M.W. Himmelstein, S.C. Carpenter, M.V. Evans, P.M. Hinderliter, E.M. Kenyon, Kinetic modeling of beta-chloroprene metabolism. II. The application of physiologically based modeling for cancer dose response analysis, Toxicol. Sci. 79 (1) (2004) 28-37; M.W. Himmelstein, R. Leonard, R. Valentine, Kinetic modeling of $\beta$-chloroprene metabolism: default and physiologically-based modeling approaches for cancer dose response, in: IISRP Symposium on Evaluation of Butadiene \& Chloroprene Health Effects, September 21, 2005, TBD—reference in this proceedings issue of Chemical-Biological Interactions] propose that observed species differences in the lung tumor dose-response result from differences in $\mathrm{CD}$ metabolic rates. The CD-PBPK model has not yet been submitted to EPA for use in developing the IRIS assessment for chloroprene, but is sufficiently developed to be considered. The process that EPA uses to evaluate PBPK models is discussed, as well as potential applications for the CD-PBPK model in an IRIS assessment.
\end{abstract}

Published by Elsevier Ireland Ltd.

Keywords: PBPK model; Chloroprene; Risk assessment; Toxicokinetic; IRIS

\footnotetext{
is International Symposium on Evaluation of Butadiene and Chloroprene Health Risks, Charleston, SC, USA, 20-22 September 2005.

动动 The views expressed in this article are those of the author and do not necessarily reflect the views or policy of the U.S. Environmental Protection Agency.

* Tel.: +1 919541 1089; fax: +1 9195410245

E-mail address: dewoskin.rob@epa.gov.
}

\section{Introduction}

The mission of the EPA to protect human health and the environment depends on the availability of quality data for quantitative characterization of the dose-response relationship. Biologically based mathe- 


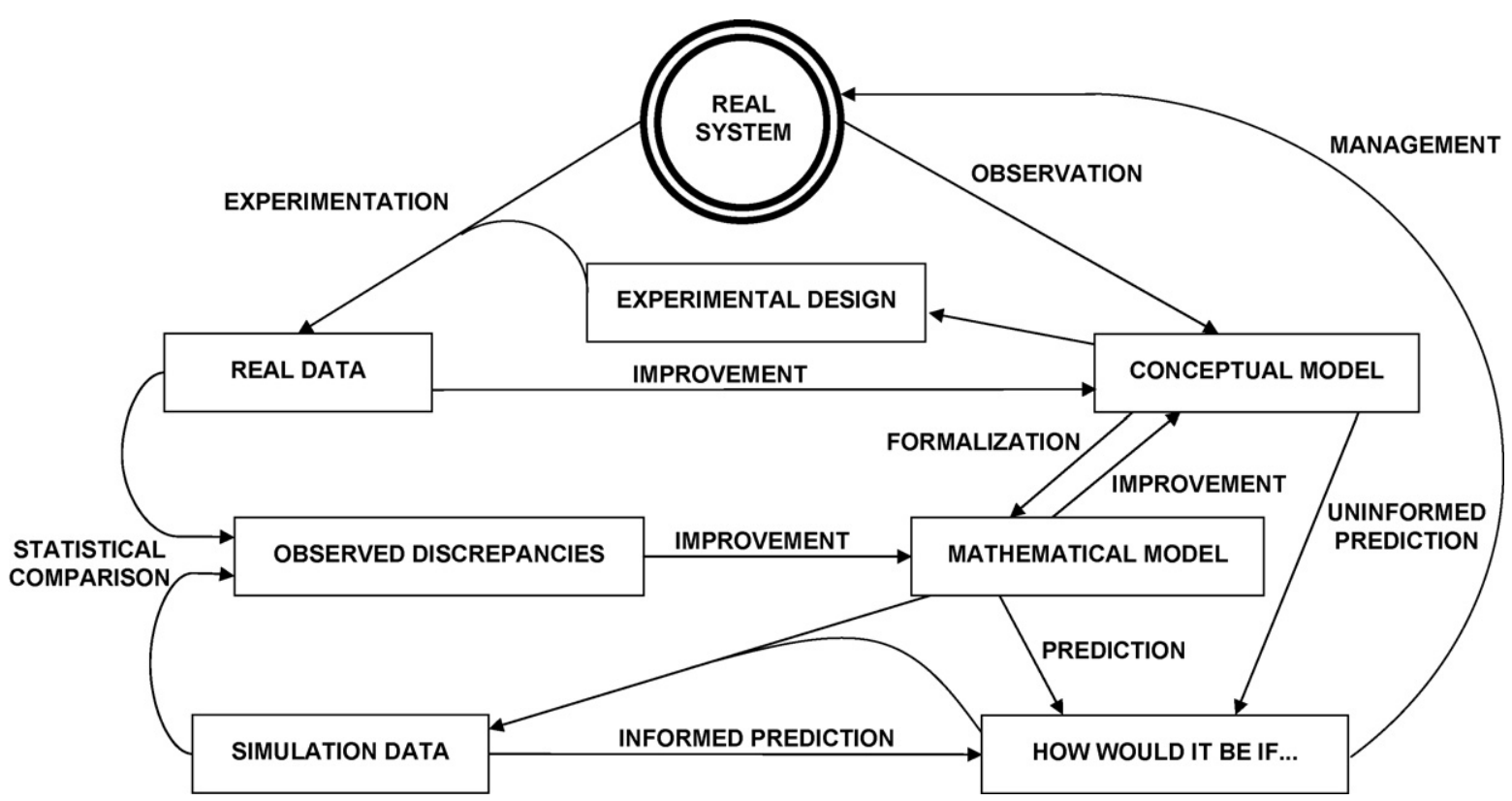

Fig. 1. The role of quantitative modeling and simulation in research is illustrated in this schematic of the relationship between a real system, a conceptualization of the system (the theoretical model), and the data. A BBDR model evolves from a qualitative (conceptual) model to a quantitative (mathematical) model of the mode of action, and therefore serves the role of both of these compartments in the figure. [Reprinted with permission from John Wiley \& Sons, Inc. from the book [1], Copyright@ 1992 Wiley-Liss, Inc.].

matical models are increasingly being used to maximize the utility of the available data for this characterization, and to identify data gaps. This presentation provides a brief overview of the development, use, and evaluation of physiologically based pharmacokinetic (PBPK ${ }^{1}$ ) models in risk assessment, and potential applications of a PBPK model for chloroprene.

\section{Development and use of PBPK models in risk assessment}

Computer models of biological systems begin with a conceptual (i.e., qualitative) representation of the components and behaviors of a real system. In an iterative process, this qualitative conception is progressively replaced with mathematical representations (i.e., the quantitative model) that simulate system behavior for comparison with experimental observations. Keen and

\footnotetext{
${ }^{1}$ Some people prefer the acronyms PBTK and PBTD, replacing "pharmacokinetic" and "pharmacodynamic" terms with "toxicokinetic" and "toxicodynamic" to distinguish models for toxic substances from those for pharmaceuticals. The Greek root "pharmakon", however, means either poison or drug, and although contemporary use associates the prefix "pharmaco-" most commonly with a medicine, both PBPK and PBTK monikers are correct, and the choice of which to use is a matter of personal preference.
}

Spain [1] illustrate this iterative process in model development in Fig. 1.

In risk assessment, the "system" behaviors to be simulated are the spatial and temporal sequence of events from the release of a chemical in the environment to an adverse effect in humans. For practical reasons, this continuous sequence is somewhat arbitrarily divided into discrete stages, each represented by a different subset of computer models, as illustrated in Fig. 2. The dosimetry models in Fig. 2 of interest here are called physiologically based pharmacokinetic (PBPK, see footnote

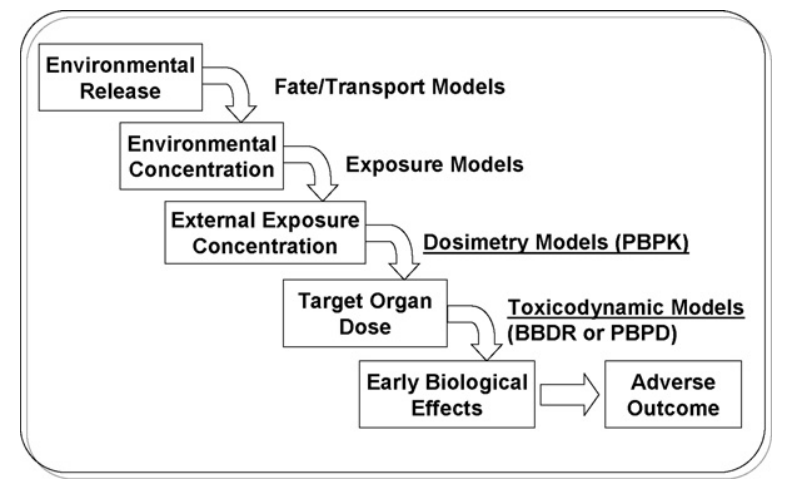

Fig. 2. Computer models for various subgroupings of the risk assessment events from release of a toxin to the environment to an adverse effect in humans. 
1) models and the dynamic models, biologically based dose-response (BBDR) models (also known as physiologically based pharmacodynamic (PBPD, see footnote 1) models) [2]. PBPK models simulate events beginning with the absorption of the external dose and ending with an internal level of a chemical at a tissue or target site (the internal dose). The "PB" in PBPK means that the model attempts to represent real physiological processes and biological tissues. The "PK" refers to the pharmacokinetic processes by which the body affects a chemical's internal disposition. BBDR models simulate the downstream events from the internal dose at a target site to the chemical's adverse effect(s) on the body, i.e., how the chemical affects the body. Both PBPK and BBDR models provide useful conceptual frameworks to organize the extant kinetic and toxicity data, and to facilitate identification of the "mode of action" (MOA) [3]. The MOA is a description of the key events (as in critical path) at the relevant level of biological organization (generally at the cell or molecular level) that leads to the adverse effects. The MOA is a subset of the complete network of all associated physiological and biochemical events.

Generally, a PBPK model can estimate many different measures of the internal dose or "dose metric" (e.g., average blood concentration, peak tissue concentration, total amount metabolized). The choice of which dose metric to use in a risk assessment is determined by the proposed MOA. Conversely, insights into the MOA are often obtained by comparing how closely different PBPK model dose metrics correlate to the adverse response(s).

Common applications of PBPK models in risk assessment are illustrated in Fig. 3, and include: (1) predicting the internal tissue level that would result from an external exposure in a test animal; (2) predicting a human equivalent internal tissue level by extrapolating from the animal kinetic data for external versus internal dose relationship; (3) predicting an animal or human internal dose level for one route of exposure (e.g., inhalation) based upon kinetic data from a different route of exposure (e.g., oral exposure); and (4) predicting variability in the human internal dose within a population due to individual differences in key physiological processes to identify sensitive sub-populations.

PBPK models require certain basic physiological data and chemical specific kinetic data for their development. The studies needed to develop a PBPK model, however, may be more cost effective and less time consuming than multiple animal bioassays (especially for assessing potential effects from inhalation exposures), and provide an ethical alternative to human dose-response studies.

For a detailed discussion and examples of PBPK model development and use in risk assessment, the reader is referred to a recent EPA report titled

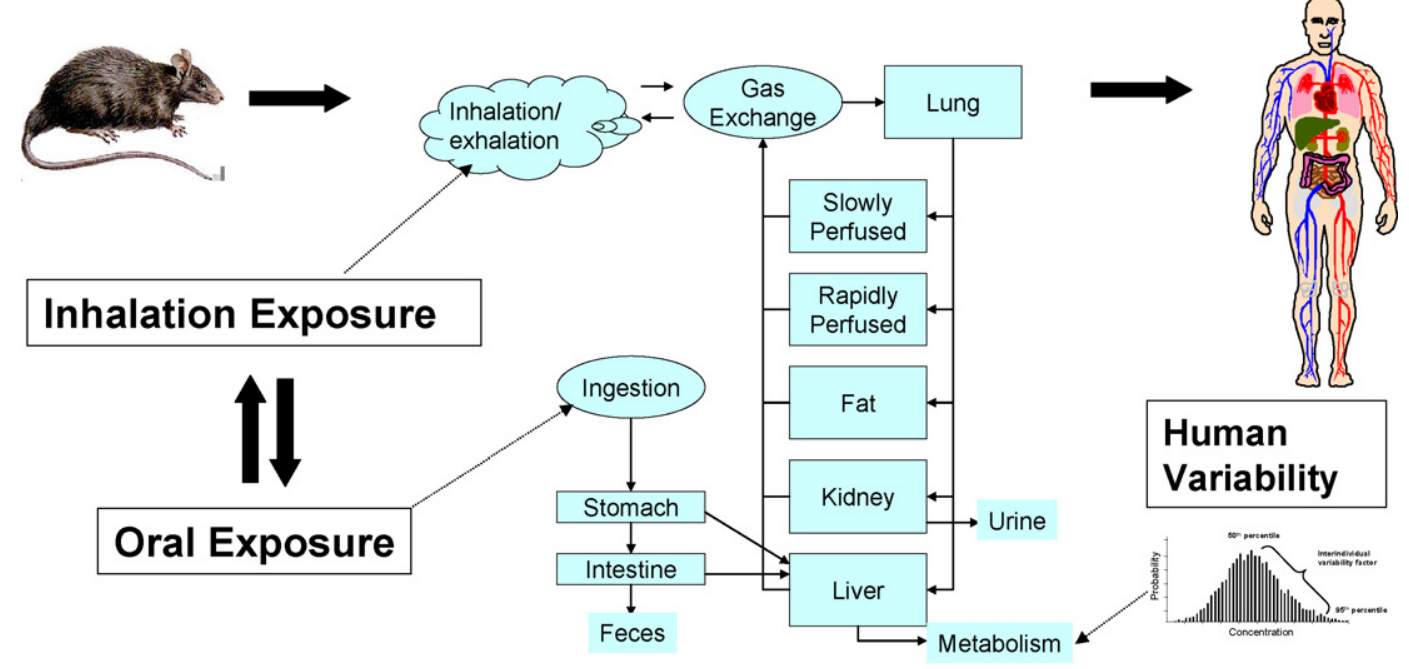

\section{- Aggregate Exposures - One chemical, multiple exposure routes \\ - Cumulative Exposure - Multiple chemicals, same or different routes \\ - Duration Extrapolation - Single dose to continuous exposure, acute $\Leftrightarrow$ subchronic $\Leftrightarrow$ chronic exposure}

Fig. 3. Sample PBPK model applications in risk assessment. 
1. Assessment of the Model Purpose

- $\quad$ Risk Assessment Needs (e.g., dose-response analysis, cumulative risks, route-to-route)

- Generic Issues (e.g., target organ, age, exposure route, species)

2. Assessment of the Biological Characterizations and Model Structure

- Consideration of Alternate Model Structures

- Appropriate Tissue Compartments

- Relevant Physiological Parameters and Biochemical Parameters

- Presence of Cross-Chemical Analogies and Chemical-Specific Characterizations

- Life-stage Considerations

- Appropriate Exposure Scenarios

3. Assessment of the Mathematical Descriptions

- Check Model Equations (e.g., unit accuracy, mass balance, and blood flow balance)

- Are Mathematical Descriptions of the Biology Reasonable

4. Assessment of the Computer Implementation

- $\quad$ Review Model Code for Proper Syntax and Mathematical Structure

- If Resources Allow, Independently Re-implement Model in Different Computer Language (or alternatively, transform model into a simpler system of differential equations and solve)

5. Parameter Analysis and Assessment of the Model Fit

- Review Parameter Documentation (model derived, literature derived, non-identifiable)

- Uncertainty in Parameters Perform

- Sensitivity Analysis

- $\quad$ Evaluate Goodness of Fit (model predictions versus data) and Statistical Analysis

6. Assessment of any Specialized Analyses

- Monte Carlo or Bayesian Methods To Quantify Uncertainty and Variability

- Parameter Co-Variance To Reflect Physiological Interdependencies

Fig. 4. Model evaluation—highlights of a six step process (with sample subheadings) for evaluating PBPK models. Adapted from [6].

"Approaches for the Application of Physiologically Based Pharmacokinetic (PBPK) Models and Supporting Data in Risk Assessment" [4].

\section{Evaluation of PBPK models used in IRIS assessments}

Quality criteria for the evaluation of PBPK models for use in risk assessment have been proposed by Clark et al. [5], and are summarized in Fig. 4. These include model purpose, biological characterization and model structure, mathematical description, computer implementation, model fit to available data, and specialized analyses (e.g., uncertainty and variability analysis, sensitivity analysis). EPA emphasizes the importance of model transparency and the availability of model code, command files (i.e., run conditions), and software. This information is needed to assure quality control for coding errors or structural flaws (such as lack of mass balance), and for peer review (external confirmation of model behavior and results). Parameter values must also be fully referenced and discussed with respect to the intended use of the model, the choices made, and alternative values. In all cases, the quality of a model depends upon the quality of the data used to calibrate and test the model for predictive capability. This is a major focus in a model evaluation.

Sensitivity analysis is used in a model evaluation to identify the parameters that have the greatest impact on a specified model output (i.e., a particular dose metric).
These results guide and support decisions on the confidence in the model predictions relative to how well supported are critical parameters with data, and which parameter estimates warrant the costs to acquire more supporting data. EPA is increasingly conducting an initial "scoping" of available PBPK models and potential uses early on in the development of a risk assessment, in large part because resource (both time and money) must be carefully planned if further model development or data are needed for a specific application. An illustration of EPA's process for model evaluation during the development of an IRIS (Integrated Risk Information System) assessment is presented in Fig. 5. IRIS is an electronic database containing information on human health effects that may result from exposure to various chemicals in the environment. EPA's process for developing IRIS assessments is available on-line [6].

Each IRIS assessment is assigned a Chemical Manager (CM) who develops the assessment and shepherds it though the multiple layers of review. EPA's National Center for Environmental Assessment (NCEA) formed a Pharmacokinetic Working Group (PKWG) to assist the CM in their evaluation of PBPK models. Fig. 5 illustrates the initial step in this evaluation (the scoping meeting) where the CM and the PKWG review the available kinetic data, proposed MOA, available models, and proposed model applications. If the model is acceptable "as is" or if resources are available to further develop the model and/or obtain needed data 


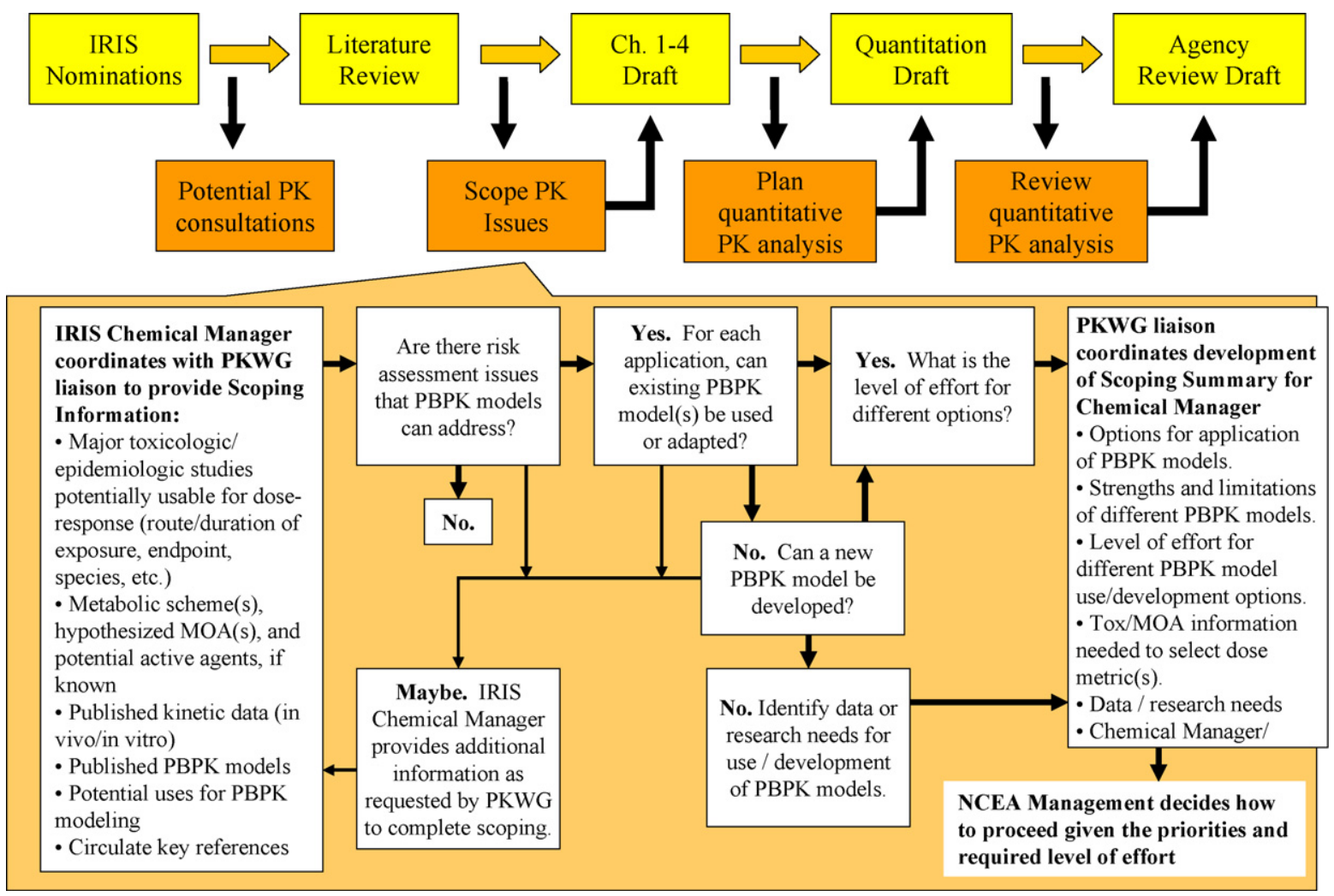

Fig. 5. PBPK model evaluation and the IRIS assessment development process-the initial scoping meeting.

for a particular application, then the model progresses through a planning, implementation, and review of its use in deriving IRIS reference values.

There are currently no general criteria to determine when a model is "good enough" for use in a risk assessment. These determinations require scientific judgment and expertise, and are made on a case-by-case basis within the context of the proposed application and the alternatives. Work continues, however, to develop criteria and guidance for model evaluation especially in key areas of model specification, calibration, and analysis as evidenced by a recent international workshop on uncertainty and variability analysis in PBPK models [7]. EPA's PKWG has also compiled an historical accounting of PBPK and classical PK models used in IRIS assessments to-date, and the impact on reference values compared with use of the default uncertainty factors [8].

\section{Chloroprene and the chloroprene PBPK model}

Chloroprene (CD), a 2-chloro analog of 1,3butadiene, is used exclusively in the production of neoprene elastomers, which are polymerized forms of polychloroprene. Polychloroprene is a synthetic rubber used in coverings or in formulations where chemical, oil, or weather resistance is needed (e.g., wire and cable covers, gaskets, adhesives, caulks, flame-resistant cushioning) $[9,10]$. CD is absorbed via inhalation, oral, and dermal routes. Parent CD is metabolized by mixed function oxidases to (1-chloroethenyl)-oxirane (1-CEO), a potentially genotoxic epoxide [11], and another metabolite, 2-chloro-2-ethenyloxirane (2-CEO). 1-CEO and 2-CEO are hydrolyzed by epoxide hydrolase, and 2-CEO is further conjugated with glutathione.

The hazardous effects from exposure to $\mathrm{CD}$ have been studied in occupational cohorts and animal bioassays [12; see I. Pagan in this issue for a discussion of CD toxicity]. Human data are limited. Animal studies report dose-response non-cancer adverse effects from inhalation exposures to $\mathrm{CD}$ consisting of lesions or functional damage to the lung, kidney, and nasal tissues. Significantly increased tumor incidence has also been observed in these and other tissues in B6C3F1 mice and F344 rats, but not in Syrian hamsters or Wistar rats.

To address the divergent responses and support extrapolation of the animal dose-response to predict the human dose-response, a PBPK model for chloro- 
prene (CD-PBPK) was developed by Himmelstein et al. $[13,14]$. The CD-PBPK model simulates disposition of chloroprene in rats, mice, hamsters, and humans following an inhalation exposure. This model has not yet been formally submitted to EPA for evaluation, so the discussion here is limited to some initial comments on the published model and its potential use in the chloroprene IRIS assessment.

The CD-PBPK model is a standard, perfusion limited model of the Ramsey and Andersen genre [15] with five compartments-liver, lung, fat, slowly and rapidly perfused tissues. CD metabolism is represented with Michaelis-Menten equations for saturable kinetics and as occurring only in the lung and liver compartments. The model estimates $\mathrm{CD}$ concentrations in each tissue compartment and blood, and is coded in the Advanced Continuous Simulation Language ( $\mathrm{ACSL}^{2}$ ).

Model parameters were developed as follows [13,14]: (1) tissue-to-blood partition coefficients were calculated from experimentally derived tissue-to-air data; (2) metabolic parameters were obtained from in vitro metabolism data for rats, mice, and humans; in vivo parameter values were scaled from in vitro data based upon the microsomal protein content in vivo; (3) liver microsomal protein content values for each species were obtained from the literature (microsomal protein content for rats was used for the hamster); (4) all species were assigned the same value for lung microsomal protein/g tissue; and (5) standard physiologic values were used to parameterize the model. In vivo gas uptake data in rats and mice were used to calibrate the model. To fit the experimental gas uptake data, only the rates of alveolar ventilation and cardiac output needed to be further adjusted. No further adjustment was needed to the other physiological and metabolic parameters obtained from in vitro experimentation.

\section{Potential applications of the CD-PBPK models in the IRIS assessment for chloroprene}

The CD-PBPK model estimates dose metrics for parent compound in blood and tissue compartments, and total amount metabolized in either the lung or liver. Based on these dose metrics, the following potential applications of the CD-PBPK model could be considered in developing the IRIS assessment for chloroprene:

1. Evaluate the mode of action by correlating parent or total amount metabolized with cancer and non-cancer endpoints.

\footnotetext{
${ }^{2}$ ACSL version 11.8.4, AEgis Technologies Group, Huntsville, AL.
}

2. Elucidate observed species differences in the external dose-response relationship.

3. Extrapolate the animal dose-response relationship to estimate the human dose-response based on the most relevant internal dose metric for the proposed MOA, the human equivalent concentration associated with a NOAEL or benchmark response level (as a point of departure), and application of any additional uncertainty factors to derive an estimate of an external exposure for a given duration to the human population (including susceptible subgroups) that is likely to be without an appreciable risk of adverse health effects over a lifetime.

4. Estimate human variability in chemical disposition within a population by using PBPK model parameter distributions (instead of point estimates) to represent variability in intra-population rates of chemical absorption, distribution, metabolism and/or elimination.

Route-to-route extrapolation of the dose-response relationship (i.e., from an inhalation route to an oral route) is not critical for chloroprene since chloroprene is highly unstable in water, and exposure and uptake by the oral route is considered unlikely.

Himmelstein et al. [16] demonstrated a greater correlation between the amount of CD metabolized in lung (mg CD metabolized/g lung/day) for each species and the tumor incidence than between external dose and tumor incidence. These results support the hypothesis (and the proposed MOA) that the observed difference between rat, mice, and hamster lung tumor dose-response are due to different rates and composition of CD metabolizing enzymes. Mice metabolize more parent $\mathrm{CD}$ than rats or hamsters, and in accordance with an MOA that proposes a metabolite as the putative toxicant, have a markedly greater incidence of lung tumors. The CD-PBPK model was then exercised with parameters set to human values (some of which were scaled from or the same as the rodent values) to predict the internal dose in humans that would yield a specified response (e.g., benchmark response level of 10\%) using the dose metric of total amount of metabolite produced in the lung, and then the external dose that would result in that level of internal dose. Himmelstein et al. [16, in this proceedings] did this work, and compared total amount metabolized in rodent and human lung and liver to the available response data from animal bioassays (tumor incidence) or human epidemiology studies (mortality rates from respiratory cancer).

Strengths in the Himmelstein et al $[13,14,16]$ CDPBPK model include: (1) the fits to the rodent in vivo gas 
uptake data using parameter estimates that were derived from in vitro studies with little need for further adjustment to parameter values; (2) the insights gained that an active metabolite is the likely toxicant as evidenced by the higher correlation between total amount metabolized in lung and lung tumor incidence compared to external dose and lung tumor incidence; and (3) support for the apparent species differences being due to differences in tissue metabolic rates.

In the full evaluation of a CD-PBPK model submitted to EPA for use in an IRIS assessment, the model assumptions and results would be reviewed in detail for scientific support of the assumptions about the metabolic pathways, the parameter values for tissue microsomal protein content in each species, and the need to reduce alveolar ventilation rate to fit the gas uptake data. The assumptions underlying the correlation of internal dose to the human lung cancer mortality data analysis will also be reviewed, but even if some of these assumptions are tenuous and the results not currently acceptable for use in an EPA assessment, these kinds of analyses expand the utility of PBPK models, and are encouraged and necessary to advance the discipline and to identify future data and research needs.

Areas of additional research (or application of the model) could include modeling the 1-CEO metabolism as a potential determinant of species differences, and addition of the kidney compartment. Data on parent or metabolite levels in vivo would obviously advance the model development and the confidence in the accuracy of the predictions, however, it is recognized that the reactivity of the parent compound and the spontaneous aqueous hydrolysis of 2-CEO $[17,18]$ confound in vivo measurement.

In conclusion, PBPK models are increasingly being used to develop quantitative dose-response relationships and reference values to protect human health. The EPA encourages the development of PBPK models for use in risk assessment, and is actively improving model evaluation and analytical tools and expertise. In its current state of development, the CD-PBPK model developed by Himmelstein et al. $[13,14,16]$ has potential for use in the development of the IRIS assessment for chloroprene based on the hypothesis that differences in metabolic rates are determinants of the observed species differences in dose-response. This hypothesis has been demonstrated for other chemicals (e.g., vinyl chloride), and PBPK models that account for the different metabolic rates have been successfully used to derive IRIS reference values [19]. Further research and development of the CD-PBPK model is encouraged to advance the understanding of the chloroprene MOA and the models use in risk assessment.

\section{References}

[1] R.E. Keen, J.D. Spain, Computer Simulation in Biology, WileyLiss, Inc., New York, USA, 1992, p. 7.

[2] US EPA, Glossary of Integrated Risk Information System (IRIS) Terms, Available at: http://www.epa.gov/iris/gloss8.htm\#b.

[3] R.S. DeWoskin, S. Barone Jr., H.J. Clewell, R.W. Setzer, Improving the development and use of biologically based dose response models (BBDR) in risk assessment, Hum. Ecol. Risk Assess. 7 (5) (2001) 1091-1120.

[4] U.S. Environmental Protection Agency, Approaches for the Application of Physiologically Based Pharmacokinetic (PBPK) Models and Supporting Data in Risk Assessment, EPA/600/R05/043F, U.S. Environmental Protection Agency, Washington, DC, 2006, Available at: http://cfpub.epa.gov/ncea/cfm/ recordisplay.cfm?deid $=135427$.

[5] L.H. Clark, R.W. Setzer, H.A. Barton, Framework for evaluation of physiologically-based pharmacokinetic models for use in safety or risk assessment, Risk Anal. 24 (6) (2004) 1697-1717.

[6] US EPA, Integrated Risk Information System (IRIS) Home Page, Available at: http://www.epa.gov/iris/index.html.

[7] US EPA, International Workshop on Uncertainty and Variability in Physiologically Based Pharmacokinetic (PBPK) Models, Research Triangle Park, NC, USA, October 31-November 2, 2006, Proceedings and white papers available at: http://www.epa. gov/comptox/uvpkm/.

[8] R.S. DeWoskin, J.C. Lipscomb, C. Thompson, W. Chiu, P. Schlosser, C. Smallwood, J. Swartout, L. Teuschler, A. Marcus, in: J.C. Lipscomb, E.V. Ohanian (Eds.), Pharmacokinetic/Physiologically Based Pharmacokinetic Models in Integrated Risk Information System Assessments, Chapter 15 in Toxicokinetics and Risk Assessment, CRC Press, ISBN: 0849337224, 2006.

[9] US Environmental Protection Agency, Health and Environmental Effects Profile for 2-Chloro-1,3-Butadiene, EPA/600/x-84/112, Environmental Criteria and Assessment Office, Office of Health and Environmental Assessment, Office of Research and Development, Cincinnati, OH, 1984.

[10] US Environmental Protection Agency, Health and Environmental Effects Document for 2-Chloro-1,3-Butadiene (Chloroprene), ECAO-CIN-G037, Environmental Criteria and Assessment Office, Office of Health and Environmental Assessment, Office of Research and Development, Cincinnati, OH, 1989.

[11] M.W. Himmelstein, N.L. Gladnick, E.M. Donner, R.D. Snyder, R. Valentine, In vitro genotoxicity testing of (1chloroethenyl)oxirane, a metabolite of beta-chloroprene, Chem. Biol. Interact. 135/136 (2001) 703-713.

[12] I. Pagan, Chloroprene: overview of studies under consideration for the development of an IRIS Assessment, in: IISRP Symposium on Evaluation of Butadiene \& Chloroprene Health Effects, September 21, 2005, TBD-reference in this proceedings issue of Chemical-Biological Interactions.

[13] M.W. Himmelstein, S.C. Carpenter, P.M. Hinderliter, Kinetic modeling of beta-chloroprene metabolism. I. In vitro rates in liver and lung tissue fractions from mice, rats, hamsters, and humans, Toxicol. Sci. 79 (1) (2004) 18-27.

[14] M.W. Himmelstein, S.C. Carpenter, M.V. Evans, P.M. Hinderliter, E.M. Kenyon, Kinetic modeling of beta-chloroprene metabolism. 
II. The application of physiologically based modeling for cancer dose response analysis, Toxicol. Sci. 79 (1) (2004) 28-37.

[15] J.C. Ramsey, M.E. Andersen, A physiologically based description of the inhalation pharmacokinetics of styrene in rats and humans, Toxicol. Appl. Pharmacol. 73 (1984) 159-175.

[16] M.W. Himmelstein, R. Leonard, R. Valentine, Kinetic modeling of $\beta$-chloroprene metabolism: default and physiologically-based modeling approaches for cancer dose response, in: IISRP Symposium on Evaluation of Butadiene \& Chloroprene Health Effects, September 21, 2005, TBD—reference in this proceedings issue of Chemical-Biological Interactions.
[17] L. Cottrell, B.T. Golding, T. Munter, W.P. Watson, In vitro metabolism of chloroprene: species differences, epoxide stereochemistry and a de-chlorination pathway, Chem. Res. Toxicol. 14 (11) (2001) 1552-1562.

[18] T. Munter, L. Cottrell, R. Ghai, B.T. Golding, W.P. Watson, The metabolism and molecular toxicology of chloroprene, Chem. Biol. Interact. (2006) (Epub ahead of print).

[19] U.S. Environmental Protection Agency, Toxicological Review of Vinyl Chloride, EPA 635/R-00/004, U.S. Environmental Protection Agency, Washington, DC, 2000, Available online at: http://www.epa.gov/iris/toxreviews/1001-tr.pdf. 